\title{
Connectivity and Performance Tradeoffs in the Cascade Correlation Learning Architecture
}

\author{
D. S. Phatak and I. Koren, Fellow, IEEE
}

\begin{abstract}
The Cascade Correlation [1] is a very flexible, efficient and fast algorithm for supervised learning. It incrementally builds the network by adding hidden units one at a time, until the desired input/output mapping is achieved. It connects all the previously installed units to the new unit being added. Consequently, each new unit in effect adds a new layer and the fan-in of the hidden and output units keeps on increasing as more units get added. The resulting structure could be hard to implement in VLSI, because the connections are irregular and the fan-in is unbounded. Moreover, the depth or the propagation delay through the resulting network is directly proportional to the number of units and can be excessive.

We have modified the algorithm to generate networks with restricted fan-in and small depth (propagation delay) by controlling the connectivity. Our results reveal that there is a tradeoff between connectivity and other performance attributes like depth, total number of independent parameters, learning time, etc. When the number of inputs or outputs is small relative to the size of the training set, a higher connectivity usually leads to faster learning, and fewer independent parameters, but it also results in unbounded fan-in and depth. Strictly layered architectures with restricted connectivity, on the other hand, need more epochs to learn and use more parameters, but generate more regular structures, with smaller, limited fan-in and significantly smaller depth (propagation delay), and may be better suited for VLSI implementations. When the number of inputs or outputs is not very small compared to the size of the training set, however, a strictly layered topology is seen to yield an overall better performance.
\end{abstract}

\section{INTRODUCTION}

A LGORITHMS like the Cascade Correlation [1] or the Continuous ID3 (CID3, which is derived from a machine learning paradigm [2]), incrementally build a neural net. These algorithms take the guess work out of the training process, there is no need to guess the number of hidden units or their connections ahead of time. The algorithms themselves add units and connections as required, during the training process. However, these algorithms can lead to unrestricted fan-in and large depth (propagation delay). Also, the connections generated are irregular and unsuitable for VLSI implementation (an efficient VLSI implementation requires regularity and local interconnects). In this paper, we propose a modified version of the Cascade Correlation algorithm which leads to

Manuscript received January 22, 1993; revised June 14, 1993. This work was supported in part by the National Science Foundation under Grant MIP 90-13013.

D.S. Phatak is with the Electrical Engineering Department, State University of New York, Binghamton, NY 13902-6000 USA,

I. Koren is with the Department of Electrical and Computer Engineering, University of Massachusetts, Amherst, MA 01003 USA

IEEE Log Number 9212061 restricted fan-in, more regular connections and significantly smaller depth.

We begin with a brief introduction to the Cascade Correlation [1]. In this method, all the inputs and outputs are directly connected first and these connections are trained to minimize the error (i.e., squared error summed over all outputs over all $\mathrm{I} / \mathrm{O}$ patterns). Hidden units are then installed one by one, incrementally reducing the error, till the desired error bound is met. Each unit is installed in two steps. In step 1, all the inputs and previously installed hidden units are connected to the input of the new hidden unit. Its output is not yet connected anywhere in the net. The input side connections of the new unit are then trained to maximize the correlation between its output and the residual error at the network outputs. This can be thought of as a maximal "alignment" with the residual error. The input side connection weights are frozen hereafter. In the second phase of installing the hidden unit, its output is connected to all the network output units. All the connections feeding the output units are then trained to minimize the error. This can be thought of as "canceling" the error as much as possible by exploiting the "alignment" accomplished in step 1. This process is continued, and new units are added until the desired error bound is met.

This turns out to be an extremely efficient, very flexible, and fast training procedure. However, each new unit in effect adds a new layer, which leads to a very deep structure with a long propagation delay. It should be noted that the presence of layer(s) skipping or shortcut connections does not reduce the propagation delay because the delay is proportional to the longest path through the network which is unaffected by the presence of shortcut connections. The propagation delay is an important performance parameter especially for high-speed applications. Moreover, learning is likely to occur infrequently and perhaps off-line. Hence, it is worthwhile to spend more time in learning if the resultant net has a much smaller propagation delay. Another drawback of the Cascade Correlation algorithm is that the fan-in of the hidden and output units keeps on increasing as more units are added. The resulting structure with irregular connections and unbounded fan-in might be hard to implement in VLSI.

Attempts have been made to reduce the connectivity [3] of the Cascade Correlation algorithm. Smotroff et al. [3] used "iterative atrophy" to prune less important connections. They came up with a saliency measure for each weight and used saliency windows to eliminate non-useful connections. Such an approach becomes computation intensive. Estimating the correct saliency for each weight can itself be a nontrivial task. 
This type of algorithm therefore spends effort first in finding all the connection weights and then in trying to undo a part of what it did and remove the non-salient connections. Another drawback of weight-pruning by saliency is that it does not lead to any improvement in the depth. In fact, the use of saliency windows would imply that the nets derived by weight pruning can be as deep as those obtained by the original version.

We have approached the problem differently and modified the Cascade Correlation algorithm to yield architectures with restricted fan-in and much smaller depth. An obvious way of controlling the fan-in and depth is to generate a "strictly layered" structure. By strictly layered, it is meant that there are no connections that skip a layer. Thus, the fan-in of a unit is limited to the number of units in the previous layer. If the number of units in each layer is sufficiently large, the number of layers needed (i.e., the depth) is usually very small as well. Next, we describe the algorithm(s) that generate the various layered topologies.

\section{The Training Procedure}

Step 1: Train the biases of the output units as best as possible, to minimize the error. Note that the inputs and outputs are not connected at this time. This is illustrated in Fig. 1(a).

Step 2: Install the first hidden layer, adding one unit at a time. Hidden units in this layer receive connections only from the input units and are not connected to other hidden units.

To install a hidden unit, it is connected to all of the network inputs and the connections are trained to maximize the correlation between its output and the residual error. Note that all the weights (input as well as output weights) associated with previously installed hidden units are held fixed when the input connections of the new unit are being trained. As a result, the new unit sees smaller residual error (at installation time) than previous units, because the previous units have already reduced the total error. The input-side weights of the new hidden unit remain frozen hereafter, as in the original Cascade Correlation algorithm.

In the second phase of installing a unit, its output is connected to all the network output units. All the fan-in connections of the output-layer units (those emanating from previously installed hidden units as well as those connected to the hidden unit being currently installed), and their biases are then trained to minimize the error.

In order to restrict the fan-in and achieve regularity, the number of units in each layer is held fixed at a predetermined value. Thus, all hidden layers (except possibly the last) have the same number of units. The creation of the first layer is illustrated in Fig. 1(b).

Step 3: Collapse the output layer into the next hidden layer. This means that the $N$ units which were output units so far, are now deemed to be the first $N$ units in the next hidden layer. The old output layer thus becomes a part of the next hidden layer. A new output layer is created and all the previous output units (which are now a part of the new hidden layer) are connected to all the new output units. These connections are trained to minimize the error. This step is depicted in Fig. 1(c).
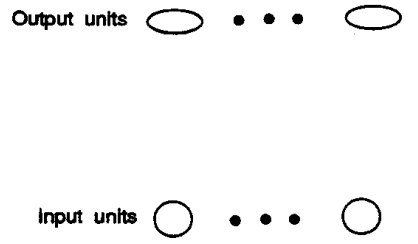

(a)

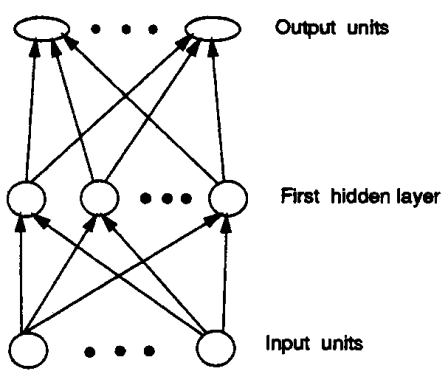

(b)

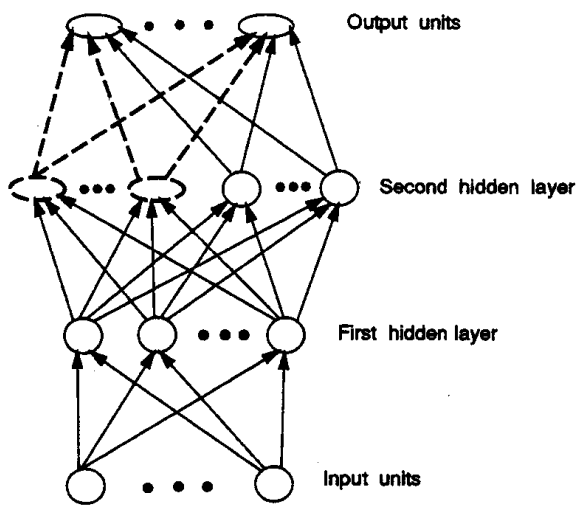

(c)

Fig. 1. (a) Step 1. Train the biases of the output units. Note that the inputs are not yet connected. (b) Step 2 . Install the first hidden layer. Units are added one at a time. Input side connections are trained first. Once trained, the input side weights remain fixed. Output side connections for the new unit are then installed and all the output connections are trained. (c) Steps 3 and 4. Install the second (and successive) hidden layers. Units in the old output layer (shown by dotted ellipses) are collapsed into the next (second) hidden layer. A new output layer is created. Input side weights of the newly collapsed hidden layer remain fixed. The output side connections of the newly formed hidden layer (shown in dotted line style) are all trained simultaneously. Then the hidden layer is further expanded by installing more units, one at a time, as in step 2 .

The motivation for utilizing the old output units and their connections in this manner is threefold. First, this reduces the training time. Second, the set of weights feeding these (previous output, now hidden) units are repeatedly trained many times, during the creation of the previous (just completed) hidden layer. Hence, the possibility of incorporating suboptimal and wasteful connections is reduced. Third, this facilitates good initial guesses of weights connecting this layer to the new output layer as explained next.

Denote the previous output units by $1,2, \cdots, i, \cdots, N$; the new output units by $1^{\prime}, 2^{\prime}, \cdots, i^{\prime}, \cdots, N^{\prime}$; and the weight of the link from unit $j$ to unit $i^{\prime}$ by $W_{j i^{\prime}}$. The biases of all the new output units are initialized to 0 . The magnitude of 
weight $W_{j i^{\prime}}$ is set proportional to the correlation between the output of unit $j$ and the target values of the $i$ th network output. If the magnitude is above a preset threshold, the sign is set positive, otherwise the sign is set negative. This way, if the $i$ th unit had produced correct outputs for most patterns, then $W_{i i^{\prime}}$ (weight of the link from unit $i$ to the corresponding output unit $i^{\prime}$ ) would be initialized to a large positive value, which would favor unit $i^{\prime}$ to turn on whenever unit $i$ is on. On the other hand, if the $i$ th unit produced wrong outputs for most patterns, then $W_{i i^{\prime}}$ would be initialized to a large negative value, which would favor unit $i^{\prime}$ to turn off whenever unit $i$ is on. Thus, this choice of initial values leads to a reduction in the output error. It was found to result in faster convergence in most cases.

Step 4: Expand the newly formed hidden layer by adding more hidden units one at a time, exactly as in step 2.

Step 5: Repeat steps 3 and 4 creating new layers until the training is successful or until a predetermined number of iterations where the trial is declared unsuccessful and is abandoned.

It should be emphasized that creating a layered topology is just one of a large number of possible ways of limiting the depth and fan-in. Even the layered topology itself can have several different variations, each of which in turn can be generated in many different ways. Indeed the Cascade Correlation is a very flexible algorithm and it is not feasible to try out all possible modifications or even a significant fraction thereof. We have tested several possibilities [4] and selected the above method for the purpose of illustration because it performs well and is relatively simple.

\section{RESUlTS AND DISCUSSION}

We have run this algorithm on a large number of problems and compared its performance to the original Cascade Correlation algorithm. For the sake of brevity, we have illustrated the results for three benchmarks namely, the Two Spirals, Vowel, and the NETTalk benchmarks from the CMU collection [5], in Tables I, II, and III, respectively. We ran 100 trials (10 sets of 10 trials each) with each algorithm, for the first two benchmarks and 10 trials with each algorithm for the NETTalk benchmark. The NETTalk nets are big and take a formidable amount of time to learn. The time required for running more than 10 trials is prohibitive. For each set, we used the same seed to initialize the random number generator in all algorithms.

Relevant parameters of interest are: maximum fan-in, number of units, total number of independent adjustable parameters (all weights and biases in the net), depth, number of epochs needed for training, number of connection crossings (please refer to [1] for definition and description of this parameter. It is a better measure of the overall learning complexity and time than the number of epochs) and the number of bit errors on the test set. These parameters were stored for each of the runs, but only the values from successful runs were used to accrue the statistics (the success rate of each of the algorithms on each of the benchmarks illustrated in the tables was $100 \%$, i.e., all the trials were successful).
TABLE I

The Two Spirals Benchmark [5], [6] With Asymmetric Sigmoidal Units (OUTPUT $\in[0,1]$ ). The PROBLEM SPECIFICATION HAS TWo INPUTS AND ONE OUTPUT

\begin{tabular}{|c|c|c|c|}
\hline \multirow{3}{*}{$\begin{array}{c}\text { Parameter } \\
1\end{array}$} & \multicolumn{3}{|c|}{ - Algorithm Type $\longrightarrow$} \\
\hline & \multirow{2}{*}{$\begin{array}{l}\text { Original } \\
\text { Cascade } \\
\text { Correlation }\end{array}$} & \multirow{2}{*}{$\frac{\frac{\text { Strictly Layered }}{\text { units per layer }}}{15}$} & \multirow{2}{*}{\begin{tabular}{|l} 
Loosely Layered \\
units per layer \\
15 \\
\end{tabular}} \\
\hline & & & \\
\hline \multirow{4}{*}{ Maximum Fan-in } & 40 & 15 & 17 \\
\hline & 25 & 15 & 17 \\
\hline & 32.2 & 15 & 17 \\
\hline & & 0 & 17 \\
\hline \multirow{4}{*}{ Number of Hidden Units } & 38 & 108 & 73 \\
\hline & 23 & 46 & 41 \\
\hline & 30 & 72 & 55 \\
\hline & 3.36 & 11.2 & 6.12 \\
\hline \multirow{4}{*}{$\begin{array}{l}\text { Total number of } \\
\text { Independent Parameters }\end{array}$} & 858 & 1537 & 1105 \\
\hline & 348 & 543 & 527 \\
\hline & 570 & 962 & 784 \\
\hline & 113.8 & 179.2 & 111.1 \\
\hline \multirow{4}{*}{ Depth } & 39 & 9 & $\overline{6}$ \\
\hline & 24 & 5 & 4 \\
\hline & 31.2 & 6.3 & 5.2 \\
\hline & 3.36 & 0.8 & 0.44 \\
\hline \multirow{4}{*}{ Number of Epochs } & 8717 & 8011 & 17643 \\
\hline & 2460 & 4008 & 3791 \\
\hline & 3337 & 5828 & 5134 \\
\hline & 768.8 & 779.3 & 1720.5 \\
\hline \multirow{4}{*}{$\begin{array}{l}\left.\text { Connection Crossings ( } \times 10^{7}\right) \\
\text { std. dev. (percentage of t }\end{array}$} & 10.4 & 15.1 & 23.7 \\
\hline & 4.04 & 5.29 & 5.48 \\
\hline & 6.78 & 9.51 & 8.1 \\
\hline & $19.87 \%$ & $18.6 \%$ & $23.9 \%$ \\
\hline \multirow{4}{*}{$\begin{array}{l}\text { Bit errors on } \\
\text { Test Set }\end{array}$} & $30(15.5 \%)$ & $27(1.3 .9 \%)$ & $17(8.8 \%)$ \\
\hline & $5(2.6 \%)$ & $4(2.1 \%)$ & $3(1.5 \%)$ \\
\hline & $15.4(7.9 \%)$ & $13.4(6.9 \%)$ & $8.9(4.6 \%)$ \\
\hline & 5.6 & 4.8 & 3.1 \\
\hline
\end{tabular}

\section{A. The Two Spirals Benchmark [5], [6]}

Table I shows the results for the Two Spirals classification problem where asymmetric sigmoidal units (output $\in(0,1)$ ) were used. The problem specification has two inputs and one output and 194 training patterns (97 points from each spiral).

Note that the table also shows a "loosely layered" version besides the strictly layered version. In the loosely layered version, all the units in a hidden layer receive connections from the original (external) inputs as well, besides the connections from the previous hidden layer. The motivation for adding the connections to the network inputs is twofold. First, note that in a strictly layered structure, the units in a layer "see" the inputs only through the previous layer. Here, it is conceivable that there could be a "loss of information" in the earlier (closer to input) layer(s) which may prevent the later (closer to output) layers from successfully learning the task. This may happen if, for example, a layer has too few units (or independent parameters) to capture all the features associated with the inputs or if certain combinations of weight values evolve during training. We encountered this situation in practice. It is intuitively clear that if the hidden units have access to the original external inputs, this kind of "irrecoverable loss of information" is not possible. Second, these connections considerably speed up the learning process, without increasing the depth, while maintaining the restricted fan-in. Note that the number of inputs is a constant. Adding connections to the inputs therefore increases the maximum fan-in by a constant amount. The fan-in is still independent of the number of hidden units installed, and is not unbounded as in the original version.

We used 15 units per hidden layer in this version. As mentioned above, the number of units (parameters) in a layer should be large enough to capture all the features of the inputs, otherwise there could be a loss of information leading to an 
TABLE II

The Vowel Benchmark [5], [7] with Symmetric Sigmoidal Units coutPuT $\epsilon[-0.5,+0.5])$. The Problem Specification has 10 InPUTS and 11 OUtPuts

\begin{tabular}{|c|c|c|}
\hline \multirow[b]{2}{*}{$\begin{array}{c}\text { Parameter } \\
1\end{array}$} & \multicolumn{2}{|c|}{$\leftarrow$ Algorithm Type $\rightarrow$} \\
\hline & $\begin{array}{l}\text { Original Cascade } \\
\text { Correlation }\end{array}$ & $\begin{array}{l}\text { Strictly Layered } \\
\text { Version }\end{array}$ \\
\hline \multirow{4}{*}{$\begin{array}{l}\text { Maximum Fan-in (Dominated } \\
\text { by number of input units }=10 \text { ) }\end{array}$} & 12 & 10 \\
\hline & 12 & 10 \\
\hline & 12 & 10 \\
\hline & 0 & \\
\hline \multirow{4}{*}{$\begin{array}{l}\text { Number of Hidden Units } \\
\text { (all in a single layer } \\
\text { for the layered version) }\end{array}$} & 1 & 6 \\
\hline & 1 & 4 \\
\hline & 1 & 4.84 \\
\hline & 0 & 0.42 \\
\hline \multirow{4}{*}{$\begin{array}{l}\text { Total number of } \\
\text { Independent Parameters }\end{array}$} & 143 & 143 \\
\hline & 143 & 99 \\
\hline & 143 & 117 \\
\hline & 0 & 9.23 \\
\hline \multirow{4}{*}{ Depth } & 2 & 2 \\
\hline & 2 & 2 \\
\hline & 2 & 2 \\
\hline & 0 & 0 \\
\hline \multirow{4}{*}{ Number of Epochs } & 406 & 579 \\
\hline & 202 & 274 \\
\hline & 287 & 423 \\
\hline & 36.96 & 55.88 \\
\hline \multirow{3}{*}{ Connection Crossings $\left(\times 10^{6}\right)$} & 2.34 & 1.82 \\
\hline & 1.11 & 0.88 \\
\hline & 1.61 & 1.36 \\
\hline std. dev. (percentage of the mean) & $13.5 \%$ & $13.2 \%$ \\
\hline \multirow{4}{*}{$\begin{array}{l}\text { Bit errors on } \\
\text { Test Set }\end{array}$} & $166(31.4 \%)$ & $100(18.9 \%)$ \\
\hline & $143(27.1 \%)$ & $69(13.1 \%)$ \\
\hline & $158.8(30.1 \%)$ & $80.8(15.3 \%)$ \\
\hline & 4.4 & 6.0 \\
\hline
\end{tabular}

unsuccessful run. After some trial and error, it was found that the strictly layered algorithm can converge if the number of units per layer is larger than 5 . At this extreme, (5 units per layer) the algorithm uses too many layers and defeats the purpose of trying to limit the depth. On the other hand, we wanted to limit the maximum fan-in to about half that of the nets generated by the original version. It was therefore decided to use 15 units per layer.

As seen in the table, the maximum fan-in (average value accrued over 100 trials) for the original Cascade Correlation algorithm is 32.2 ; while that for the strictly layered version is 15; and for the loosely layered version, it is 17 . Thus, the fanin is reduced by about one-half. Similarly, the depths (average) for the layered versions are 6.3 and 5.2 while the depth generated by the original version is 31.2 . This demonstrates a significant reduction in the depth or the propagation delay (about 5 times smaller depth). The number of units used by the layered versions, on the other hand, increases by a factor between 2 and 2.5. However, the total number of independent parameters (weights and biases) is a better measure of the overall complexity than the number of units alone, because it incorporates both the number of units and their connectivity. It is seen that the strictly layered version uses about 1.6 times; and the loosely layered version uses about 1.37 times the number of parameters used by the original algorithm.

Cios and Liu [2] have also used the two spirals benchmark to illustrate their CID3 algorithm. The net reported in [2] has 30 hidden units distributed in FIVE hidden layers (depth $=6$ ), a maximum fan-in of 32 , and requires 431 independent parameters. Thus, the number of parameters utilized by CID3 is smaller than that utilized by the loosely layered version (527), but the maximum fan-in is about double that of the loosely layered version (17). The depths of the generated nets are comparable. In fact, the CID3 has some of the same drawbacks as the Cascade Correlation algorithm: it connects all previously installed hidden layers to the new layer being created, which leads to an unbounded fan-in and irregular connections.

The spirals used to generate Table I had a radius $R=2.0$ and were centered at $(2,2)$ in the $x y$ plane. Consequently, they were contained in the square with vertices $\{(0,0),(0,4),(4,4)$, $(4,0)\}$ which lies entirely in the first quadrant. The net reported in [2], on the other hand, was trained on spirals centered at the origin. For a proper comparison with CID3, we therefore used spirals centered at the origin $(0,0)$ with a radius $R=6.5$. This way the $x$ and $y$ coordinates of the points on the spirals take both positive and negative values and are symmetric with respect to the origin. For this training data, the loosely layered version of our algorithm generated nets with an average of six hidden layers (depth $=7$ ), with seven hidden units per layer, and utilized 436 independent parameters on the average (the table corresponding to this data had to be excluded from the manuscript for the sake of brevity). These nets are better than the net generated by the CID3 algorithm since the depth and number of independent parameters used is about the same, but the maximum fan-in is restricted to the fixed value 9 (about one-third), and the connections are more regular.

\section{B. The Vowel Benchmark [5], [7]}

Table II shows the results for the Vowel Benchmark with symmetric sigmoidal units (output $\in[-0.5,+0.5]$ ). The net has 10 inputs and 11 outputs, where exactly one out of the 11 output units is "on" at a time, to indicate the spoken vowel. This corresponds to a localized output encoding. As seen in the table, the number of hidden units employed is usually very small (in fact, smaller than the number of input or output units). The maximum fan-in is therefore dominated by the number of input connections. Just one hidden layer with a small number of hidden units was found to be sufficient for this task. Hence, is not necessary to try the loosely layered version. Note that the total number of parameters (average value) used by the layered version is significantly smaller than that of the original version.

\section{The NETTalk Benchmark [5], [8]}

Table III shows the results for this benchmark for which there are 196 input and 26 output units. We randomly chose a set of 200 words (from the 1000 available in the CMU version), which generated 1114 training patterns. Here the fanin is dominated by the number of input units. It interesting to note that the strictly layered version uses a much larger number of hidden units but a smaller total number of independent parameters. This is due to the restricted connectivity. Also, the depth is much smaller than that generated by the original version (about one-sixth). An examination of the nets revealed that the direct input-output connections in the original version is the single most dominant contributor to the total number of independent parameters $(196 \times 26=5096$ or about $50 \%$ of the total number of parameters. Please refer to Table III). Since the strictly layered version performs better without these connections, there is no need to try the loosely layered version where these connections are present. 
TABLE III

The NETTalk Benchmark [5], [8] with Symmetric SigmoIDAL UnITS. THERE ARE 196 INPUTS AND 26 OUTPUTS

\begin{tabular}{|c|c|c|}
\hline \multirow[b]{2}{*}{$\begin{array}{c}\text { Parameter } \\
\downarrow\end{array}$} & \multicolumn{2}{|c|}{-Algorithm Type $\longrightarrow$} \\
\hline & $\begin{array}{c}\text { Original Cascade } \\
\text { Correlation } \\
\end{array}$ & $\begin{array}{l}\text { Strictly Layered Version } \\
\text { (35 units per layer) }\end{array}$ \\
\hline \multirow{4}{*}{$\begin{array}{l}\text { Maximum Fan-in (Dominated } \\
\text { by number of input units = 196) }\end{array}$} & 2226 & 196 \\
\hline & 221 & 196 \\
\hline & 223 & 196 \\
\hline & 1.49 & 0.0 \\
\hline \multirow{4}{*}{ Number of Hidden Units } & 29 & 132 \\
\hline & 24 & 100 \\
\hline & 25.7 & 104.8 \\
\hline & 1.49 & 8.71 \\
\hline \multirow{4}{*}{$\begin{array}{l}\text { Total number of } \\
\text { Independent Parameters }\end{array}$} & 11995 & 11115 \\
\hline & 10750 & 10041 \\
\hline & 11172 & 10265 \\
\hline & 371.9 & 285.8 \\
\hline \multirow{4}{*}{ Depth } & 30 & 5 \\
\hline & 25 & 4 \\
\hline & 26.70 & 4.08 \\
\hline & 1.49 & 0.29 \\
\hline \multirow{4}{*}{ Number of Epochs } & 5080 & 5812 \\
\hline & 4340 & 5300 \\
\hline & 4641 & 5565 \\
\hline & 194.9 & 179.4 \\
\hline \multirow{4}{*}{ Connection Crossings ( $\left.\times 10^{9}\right)$} & 21.3 & 6.84 \\
\hline & 17.5 & 6.43 \\
\hline & 19.1 & 6.64 \\
\hline & $5.6 \%$ & $2 \%$ \\
\hline
\end{tabular}

\section{Trade-offs}

Our results indicate that the layered version always requires more hidden units. This is expected, since the unit being installed has fewer connections. It can only see units in the previous hidden layer and thus has less information to work with than a unit in the original version, which is connected to all the previous units and network inputs.

The number of independent parameters, which is a better measure of the overall network complexity, however, shows an interesting behavior. When the number of training patterns is large compared to the number of inputs or outputs, the original version utilizes fewer parameters than the layered version. This is illustrated by the two spirals benchmark where the number of input units is two, and the number of $\mathrm{I} / \mathrm{O}$ pairs to be learned is 194 or about 100 times larger. For such problems, there is a tradeoff between connectivity and other performance attributes. The original version with full connectivity is at one extreme. It yields faster convergence and a smaller number of parameters but also leads to very deep nets with arbitrarily large fan-in. The strictly layered version is at the other extreme and requires more parameters and longer learning time, but yields restricted fan-in and much smaller depth. The loosely layered version falls in between these extremes, trading off more connectivity for fewer parameters and equal or smaller depth, and may be the best compromise.

When the input or output dimensionality is not so small compared with the size of the training set, the layered version utilizes fewer parameters and yields overall better performance. This is illustrated by the Vowel and the NETTalk benchmarks. The ratio of the number of training patterns to the number of input units is about 9 and 5, for the Vowel and NETTalk benchmarks, respectively. In such cases, connecting the network inputs to the outputs results in a large (in fact larger than required) number of connections. Furthermore, training all of them simultaneously turns out to be less efficient than incrementally introducing hidden units and training the small number of associated connections at a time.
In conclusion, we have proposed a modified version of the Cascade Correlation algorithm which controls the connectivity of the units being added to restrict the fan-in and generates layered nets with a very small depth and regular connections. Our data illustrates the tradeoffs between connectivity and other performance attributes.

Several future extensions are possible. For instance, the number of units per layer need not be fixed. It can be decided dynamically and the creation of a new layer can be started if the error reduction achieved by expanding the layer begins to taper off. Another approach is to stick to the strictly layered structure as far as possible and only occasionally install units with direct (layers skipping) connections to the network inputs, when there is little or no reduction in the error.

Preliminary analysis of the generalization characteristics of these nets has been done. Even though the layered version utilizes a larger number of parameters, its generalization performance appears to be comparable to or better than the original version, as illustrated by the number of bit errors on the test sets for the Spirals and Vowel benchmarks in Tables I and II. However, further investigation is necessary to draw any conclusions.

\section{REFERENCES}

[1] S. E. Fahlman and C. Lebiere, "The cascade correlation learning architecture," in Neural Information Processing Systems 2, D. S. Touretzsky, ed. Morgan Kaufman, 1990, pp. 524-532.

[2] K. J. Cios and N. Liu, "A machine learning method for generation of a neural network architecture: A continuous ID3 algorithm," IEEE Trans. Neural Net., vol. 3, pp. 280-291, Mar. 1992

[3] I. G. Smotroff, D. H. Friedman, and D. Connolly, "Large scale networks via self organizing hierarchical networks," in Proc. SPIE Conf. Appl. AI and Neural Net., Apr. 1991.

[4] D. S. Phatak and I. Koren, "Connectivity and performance tradeoffs in the cascade correlation learning architecture," Tech. Rep. TR-92-CSE27, Electrical and Computer Engineering Dept., Univ. of Massachusetts, Amherst, July 1992.

[5] S. E. Fahlman, et al., "Neural nets leaming algorithms and benchmarks database," maintained by S. E. Fahlman, et al. at the Computer Science Dept., Carnegie Mellon University.

[6] K. J. Lang and M. J. Witbrock, "Learning to tell two spirals apart," in Proc. 1988 Connectionist Models Summer School, San Mateo, CA, 1988.

[7] A. J. Robinson and F. Fallside, "A dynamic connectionist model for phoneme recognition," in Proc. Euro, Paris, France, June 1988.

[8] T. J. Sejnowski and C. R. Rosenberg, "Parallel networks that learn to pronounce English text," Complex Systems, vol. 1, pp. 145-168, 1987.

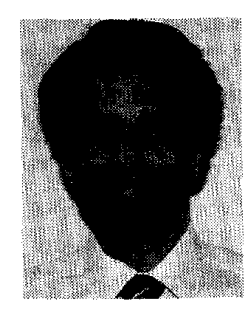

D. S. Phatak received the B. Tech. degree in Electrical Engineering from the Indian Institute of Technology, Bombay, in 1985, the M.S. in Microwave Engineering in 1990 and the Ph.D. in Computer Systems Engineering in 1993, both from the Electrical and Computer Engineering Department, University of Massachusetts, Amherst.

He recently became Assistant Professor of Electrical Engineering at the State University of New York, Binghamton. His current research interests are in theory, applications and electronic as well as optical implementations of neural networks, digital and analog VLSI design and $\mathrm{CAD}$, fault tolerant computing, computer arithmetic algorithms and their VLSI implementations. Dr. Phatak has published in the IEEE Transactions on Neural Networks, Neural Computation, IEEE Transactions on Computers, IEEE Transactions on Microwave Theory and Techniques, and in conferences related to signal processing algorithms and their implementations. 


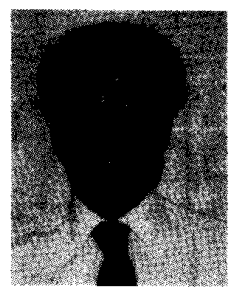

I. Koren (S'72-M'75-SM'87-F'91) received the B.Sc., M. Sc. and D. Sc. degrees from the TechnionIsrael Institute of Technology, Haifa, in 1967, 1970, and 1975, respectively, all in electrical engineering. $\mathrm{He}$ is currently a Professor of Electrical and Computer Engineering at the University of Massachusetts, Amherst. Previously he was with the Departments of Electrical Engineering and Computer Science at the Technion-Israel Institute of Technology. He also held visiting positions with the University of California at Berkeley, University of Southern California, Los Angeles and University of California, Santa Barbara. He has been a consultant to several companies including Intel, Digital Equipment Corp., National Semiconductor and Tolerant Systems.

Dr. Koren's current research interests are fault-tolerant VLSI architectures, models for yield and performance, floor-planning of VLSI chips and computer arithmetic. He was a co-guest editor for the IEEE Transactions on Computers, special issue on High Yield VLSI Systems, April 1989. Since January 1992, he serves on the Editorial Board of these Transactions. He also served as Program Committee member for numerous conferences. He has edited and co-authored the book, Defect and Fault-Tolerance in VLSI Systems, Vol. 1 (Plenum, 1989). He is the author of the textbook Computer Arithmetic Algorithms, (Prentice-Hall, 1993). 